\title{
Aplicação clínica da ultrassonografia craniana com Doppler em neonatos prematuros de muito baixo peso*
}

\author{
Clinical application of transcranial Doppler ultrasonography in premature, very-low-birth-weight \\ neonates
}

Marta Lúcia Gabriel ${ }^{1}$, Vânia Belintani Piatto², Antônio Soares Souza ${ }^{3}$

Resumo OBJETIVO: Analisar o valor do diagnóstico precoce de alterações hemodinâmicas em hemorragias e eventos hipóxico-isquêmicos pela avaliação de imagens e mensuração do índice de resistência por meio da ultrassonografia craniana com Doppler em neonatos prematuros de muito baixo peso. MATERIAIS E MÉTODOS: Cinquenta neonatos prematuros de muito baixo peso foram submetidos a ultrassonografia craniana com Doppler com a técnica transfontanela anterior e transtemporal sequenciais. RESULTADOS: Foram detectadas alterações cerebrais em $32 \%$ dos prematuros, sendo $22 \%$ com hemorragia intracraniana, $8 \%$ com leucomalácia periventricular e $2 \%$ com toxoplasmose. Dentre os 34 casos $(68 \%)$, do total de neonatos, nos quais não foram detectadas lesões cerebrais pela ultrassonografia craniana, 18 (53\%) apresentaram alterações no índice de resistência. $O$ índice de resistência variou conforme a época do exame. CONCLUSÃO: Existe correlação entre a presença de alterações na hemodinâmica cerebral e subsequente desenvolvimento de hemorragias e lesões hipóxico-isquêmicas, pela mensuração do índice de resistência. Alterações do índice de resistência, embora não preditoras de morte, estão relacionadas com a gravidade do quadro clínico em neonatos prematuros de muito baixo peso.

Unitermos: Doppler transcraniano; Prematuros de muito baixo peso; Ultrassom; Índice de resistência.

Abstract OBJECTIVE: The present study was aimed at analyzing the value of the early diagnosis of hemodynamic changes in hemorrhages and hypoxic-ischemic events in premature, very-low-birth-weight neonates through the evaluation of images and resistance index measurement by means of transcranial Doppler ultrasonography. MATERIALS AND METHODS: Fifty premature, very-low-birth-weight neonates were submitted to transcranial Doppler ultrasonography with sequential transfontanellar and transtemporal techniques. RESULTS: Cerebral abnormalities were detected in $32 \%$ of the neonates $122 \%$ with intracranial hemorrhage, $8 \%$ with periventricular leukomalacia, and $2 \%$ with toxoplasmosis). Among the 34 cases $(68 \%)$ of neonates in whom no brain lesion was detected at transcranial Doppler ultrasonography, $18(53 \%)$ presented changes in the resistance index. Such resistance index varied according to the time of the examination. CONCLUSION: There is a correlation between the presence of cerebral hemodynamic changes demonstrated by resistance index measurements and the subsequent development of hemorrhages and hypoxic-ischemic lesions. Although not being a death predictor, changes in the resistance index are associated with the severity of the clinical conditions in preterm, very-low-birth-weight neonates.

Keywords: Transcranial Doppler ultrasonography; Premature, very-low-birth-weight neonates; Ultrasonography; Resistance index.

Gabriel ML, Piatto VB, Souza AS. Aplicação clínica da ultrassonografia craniana com Doppler em neonatos prematuros de muito baixo peso. Radiol Bras. 2010;43(4):213-218.

* Trabalho realizado no Hospital de Base - Fundação Faculdade Regional de Medicina (Funfarme), São José do Rio Preto, SP, Brasil.

1. Mestre, Médica Radiologista do Hospital de Base - Fundação Faculdade Regional de Medicina (Funfarme), São José do Rio Preto, SP, Brasil.

2. Doutora, Professor Adjunto IV-D da Faculdade de Medicina de São José do Rio Preto (Famerp), São José do Rio Preto, SP, Brasil.

3. Professor Doutor, Médico Radiologista Pediátrico do Departamento de Imagem da Faculdade de Medicina de São José do Rio Preto (Famerp), São José do Rio Preto, SP, Brasil.

Endereço para correspondência: Dra. Marta Lúcia Gabriel. Avenida Brigadeiro Faria Lima, 5416, Vila São Pedro. São José do Rio Preto, SP, Brasil, 15090-000. E-mail: depimagem@ famerp.br

Recebido para publicação em 3/11/2009. Aceito, após revisão, em 21/6/2010.

\section{INTRODUÇÃO}

Os avanços tecnológicos e científicos levaram à obtenção de resultados satisfatórios na assistência neonatal, com significativa melhora nos cuidados intensivos aos recém-nascidos prematuros, possibilitando maior sobrevida destes, e com isso permitindo intervenções obstétricas precoces $^{(1)}$.

A hemorragia intracraniana (HIC) e a leucomalácia periventricular (LPV) são as afecções cerebrais mais comuns do recém- nascido, sendo os prematuros os mais acometidos $^{(2,3)}$. Essas afecções são de causas multifatoriais, nas quais estão envolvidos fatores vasculares, hemodinâmicos, inflamatórios e infecciosos, podendo causar sequelas neuropsicomotoras e levar a paralisia cerebral e/ou déficit cognitivo e comportamental $^{(4)}$.

Em neonatos prematuros, a HIC é mais frequente nas regiões subependimária, intraventricular e/ou intraparenquimatosa, enquanto no neonato a termo a HIC é rara e, quando ocorre, os espaços subdural e 
subaracnoide são os locais mais prevalentes. Por outro lado, a localização mais comum na LPV inclui a substância branca peritrigonal occipital adjacente ao forame interventricular, na coroa radiada, onde se encontram as artérias ventriculopetais e ventriculofugais, consideradas as áreas de fronteira ou áreas de irrigação no cérebro do recém-nascido prematuro ${ }^{(3)}$.

A gravidade da HIC em lactentes prematuros pode ser avaliada de acordo com a classificação de Papile et al., que é o sistema mais usado. Essa classificação foi estabelecida com base na localização topográfica da hemorragia e na presença de dilatação ventricular, sendo considerados os graus I e II leve, III moderado e IV grave ${ }^{(\mathbf{5})}$.

A fim de uma melhor conduta durante o tratamento intensivo do prematuro, o método diagnóstico mais indicado é a ultrassonografia craniana (USC) com Doppler. O ultrassom possibilita o diagnóstico por imagem e o Doppler fornece informações relativas à hemodinâmica cerebral pela análise dos principais ramos do polígono arterial de Willis. Além disso, o Doppler, por ser feito via transfontanela anterior e temporal, permite melhor a visibilização dos vasos sanguíneos, possibilitando a quantificação das variações do fluxo sanguíneo cerebral em determinado período de tempo e a mensuração do índice de resistência (IR) por meio da representação espectral da onda ${ }^{(6)}$.

O IR é definido pela equação:

$$
\mathrm{IR}=\mathrm{S}-\mathrm{D} / \mathrm{S},
$$

sendo: S a velocidade do fluxo sistólico, D a velocidade do fluxo diastólico, com valores de referência entre 0,60 e 0,80 , fornecendo informações se houve alteração hemodinâmica em hemorragias e eventos hipóxico-isquêmicos ${ }^{(6,7)}$.

O Doppler tem sido extensivamente realizado em prematuros para avaliar as mudanças na hemodinâmica cerebral ${ }^{(\mathbf{8})}$, e estudos descritos na literatura têm confirmado que essas alterações estão relacionadas aos mecanismos fisiopatológicos das hemorragias e eventos hipóxico-isquêmi$\cos ^{(8)}$. Entretanto, ainda existem dúvidas em relação se o Doppler utilizado para monitorar as alterações hemodinâmicas cerebrais pode ser de valor para o diagnóstico precoce e predizer o prognóstico das hemorragias e eventos hipóxico-isquêmicos no período neonatal ${ }^{(8)}$.

Diante do exposto, este estudo teve como objetivos analisar o valor do diagnóstico precoce de alterações hemodinâmicas em hemorragias e eventos hipóxico-isquêmicos pela avaliação de imagens e mensuração do IR por meio da USC com Doppler, a fim de avaliar o prognóstico, considerando gravidade e óbito.

\section{MATERIAIS E MÉTODOS}

No período de janeiro a setembro de 2008 foram estudados, prospectivamente, 50 neonatos prematuros de muito baixo peso ( $<32$ semanas de gestação, $<1.500 \mathrm{~g}$ ), sendo 24 do gênero masculino e 26 do gênero feminino, independente da etnia.

Os recém-nascidos foram examinados na Unidade de Terapia Intensiva Neonatal e na Unidade de Ultrassonografia do Hospital-Escola da Instituição. Os pais e/ou responsáveis foram informados sobre $\mathrm{o}$ estudo mediante termo de consentimento, após aprovação do Comitê de Ética em Pesquisa, sob parecer $n^{\circ} 200 / 2004$.

Os exames ultrassonográficos foram realizados em equipamentos ATL-HDI 3000 e ATL-HDI 5000 Sono CT, ambos fabricados pela Philips Medical Systems (Bothell, WA, EUA), utilizando-se transdutor de alta frequência convexo de $8,5 \mathrm{MHz}$ e Doppler colorido.

Os neonatos foram submetidos a quatro USCs pela técnica transfontanela anterior e transtemporal ${ }^{(\mathbf{9 , 1 0})}$. Estes exames foram realizados de acordo com o Protocolo do Serviço de Ultrassonografia da Instituição, o qual é dividido nos seguintes períodos: período 1: 1 a 5 dias de vida; período 2: 10 a 17 dias de vida; período 3: 18 a 30 dias de vida; período 4: 31 a 44 dias de vida.

Em razão de as alterações cerebrais ocorrerem nos primeiros dias de vida, as USCs foram realizadas precocemente, do primeiro ao quinto dia de vida. No período de 6 a 9 dias de vida, não foi realizada a USC, para estabilização clínica do neonato, procedendo-se novas USCs nos períodos seguintes para monitoramento da lesão.

Os exames foram realizados em cortes nos planos coronal (anterior, médio e posterior), sagital (mediano e paramediano) e axial. No corte coronal anterior foram ob- tidas imagens de placa crivosa do etmoide, órbitas, parênquima frontal e cornos anteriores dos ventrículos laterais. No corte coronal médio foram observados fissura inter-hemisférica, corpo caloso, cavum do septo pelúcido, forames de Monro, terceiro ventrículo, plexos coroides dos ventrículos, matriz germinativa, cabeça do núcleo caudado, tálamos, fissura de Sylvius, ínsula, globo pálido e putâmen e regiões parietais e temporais do parênquima cerebral. Pelo corte coronal posterior foram obtidas imagens da região atrial dos ventrículos laterais, plexos coroides, cornos occipitais dos ventrículos laterais, região posterior ao átrio dos ventrículos laterais, cerebelo, cisterna magna e lobo occipital. No corte sagital mediano foram obtidas imagens da fissura inter-hemisférica, corpo caloso, cavum do septo pelúcido, terceiro ventrículo, quarto ventrículo e cisterna magna, aqueduto cerebral, vermis cerebelar, ponte e bulbo. No corte sagital paramediano foram obtidas imagens dos cornos anteriores, temporais e occipitais dos ventrículos laterais, líquor cefalorraquidiano, plexos coroides dos ventrículos laterais, matriz germinativa, tálamos, hipotálamos e núcleos da base, fissura de Sylvius, regiões temporal, parietal e occipital do parênquima cerebral.

Com auxílio do Doppler foram estudadas a anatomia e vasculatura cerebral, pela análise do IR, nas seguintes artérias: cerebral anterior, média e posterior. Além disso, foi realizada a quantificação das variações relativas do fluxo sanguíneo cerebral em determinado período de tempo e a mensuração do IR. O IR foi calculado por meio da fórmula:

$$
\mathrm{IR}=\mathrm{S}-\mathrm{D} / \mathrm{S},
$$

onde: $\mathrm{S}$ corresponde à velocidade do fluxo sistólico e D, à velocidade do fluxo diastólico. Neste estudo foram considerados como IR normal na artéria cerebral os valores entre 0,60 e $0,80^{(6,7)}$.

Os dados foram avaliados empregandose estatística descritiva e análises comparativas. Para avaliar a diferença entre os valores médios do IR das artérias cerebrais (anterior, média e posterior) dos neonatos prematuros sem alterações cerebrais e com HIC submetidos à ultrassonografia nos quatro períodos de exames realizados, foi aplicado o teste $t$ com correção de Welch ${ }^{(11)}$. 
O prognóstico (piora ou morte), segundo Doppler alterado ou não, foi obtido pelo teste qui-quadrado por análise de dependência $^{(\mathbf{1 2})}$. Foi considerado o nível de significância para valor de $p \leq 0,05$.

\section{RESULTADOS}

Foram avaliados, no período do estudo, 50 neonatos prematuros de muito baixo peso, sendo 24 (48\%) do gênero masculino e $26(52 \%)$ do gênero feminino. A idade gestacional variou de 29 a 32 semanas ( 30,8 $\pm 1,5$ semanas) e o peso ao nascimento variou entre 550 e $1.500 \mathrm{~g}(1.179 \pm 288 \mathrm{~g})$.

Em relação aos resultados das USCs realizadas nos dois primeiros períodos, foram encontrados $34(68 \%)$ neonatos sem alterações cerebrais e 16 (32\%) com alterações. Dentre os 16 neonatos com alterações cerebrais, foram encontrados 11 casos de HIC (69\%), 4 de LPV (25\%) e 1 de toxoplasmose cerebral $(6 \%)$.

O IR foi calculado em todas as USCs realizadas nos 50 neonatos do estudo, independente da presença ou não de lesões cerebrais. A Tabela 1 apresenta os valores médios do IR para as artérias cerebrais anterior, média e posterior de todos os neonatos avaliados.

Pela análise da Tabela 1 foi observado aumento gradativo nos valores médios do IR nas três artérias avaliadas nos exames realizados nos quatro períodos. Em relação ao período 1, os valores mínimo e máximo, para cada artéria analisada foram, respectivamente: na artéria cerebral anterior, 0,56 e 0,74 ; na artéria cerebral média, 0,63 e 0,85 ; e na artéria cerebral posterior, 0,6 e 0,78 , indicando que alterações no IR ocorreram já nos primeiros dias de vida.

Dos 11 casos de HIC, 5 foram classificados em grau I, 2 em grau II, 3 em grau III e 1 em grau IV. Houve alteração no valor do IR em 10 pacientes, sendo 4 casos em uma das artérias cerebrais, 2 em duas das artérias e 4 nas três artérias. $\mathrm{O}$ valor médio do IR nas artérias cerebrais anterior, média e posterior dos neonatos com HIC $(n=11)$ aumentou do primeiro ao terceiro exame, conforme demonstrado na Tabela 2.

De acordo com a Tabela 2 , no período 1 o IR médio da artéria cerebral anterior $(0,57)$ estava abaixo do limite de referência $(0,60$ a 0,80$)$, e no período 3 o IR mé-
Tabela 1 Valores de média e desvio-padrão do IR das artérias cerebrais dos neonatos prematuros $(n=$ 50) submetidos à USC com Doppler.

\begin{tabular}{ccccc}
\hline Período & Exame (dias) & ACA (M \pm DP) & ACM (M \pm DP) & ACP (M \pm DP) \\
\hline 1 & 1 a 5 & $0,65 \pm 0,09$ & $0,74 \pm 0,11$ & $0,69 \pm 0,09$ \\
2 & 10 a 17 & $0,69 \pm 0,09$ & $0,77 \pm 0,08$ & $0,73 \pm 0,09$ \\
3 & 18 a 30 & $0,72 \pm 0,07$ & $0,77 \pm 0,09$ & $0,74 \pm 0,09$ \\
4 & 30 a 44 & $0,75 \pm 0,10$ & $0,79 \pm 0,09$ & $0,76 \pm 0,07$ \\
\hline
\end{tabular}

ACA, artéria cerebral anterior; ACM, artéria cerebral média; ACP, artéria cerebral posterior; M, média; DP, desvio padrão.

Tabela 2 Valores de média e desvio-padrão do IR das artérias cerebrais dos neonatos prematuros com HIC $(n=11)$ submetidos à USC com Doppler.

\begin{tabular}{ccccc}
\hline Período & Exame (dias) & ACA (M $\pm \mathrm{DP})$ & ACM (M \pm DP) & ACP (M \pm DP) \\
\hline 1 & 1 a 5 & $0,57 \pm 0,06$ & $0,68 \pm 0,10$ & $0,64 \pm 0,07$ \\
2 & 10 a 17 & $0,63 \pm 0,16$ & $0,75 \pm 0,12$ & $0,66 \pm 0,17$ \\
3 & 18 a 30 & $0,71 \pm 0,04$ & $0,85 \pm 0,07$ & $0,78 \pm 0,10$ \\
4 & 30 a 44 & $0,74 \pm 0,11$ & $0,75 \pm 0,05$ & $0,72 \pm 0,04$ \\
\hline
\end{tabular}

ACA, artéria cerebral anterior; ACM, artéria cerebral média; ACP, artéria cerebral posterior; M, média; DP, desviopadrão. dio da artéria cerebral média $(0,85)$ estava acima do limite de referência. Mas, ainda em relação ao período 1 , os valores mínimo e máximo do IR para cada artéria foram, respectivamente: na artéria cerebral anterior, 0,51 e 0,63; na artéria cerebral média, 0,58 e 0,68 ; e na artéria cerebral posterior, 0,57 e 0,71 , significando que nestes neonatos com HIC o IR já estava alterado neste período.

Dos quatro casos com LPV, dois (50\%) apresentaram alterações no IR, sendo um caso com alterações nas três artérias cerebrais e o outro caso em duas artérias, a anterior e a média. Foram encontrados os seguintes valores mínimo e máximo do IR, respectivamente, para cada artéria cerebral avaliada: na artéria cerebral anterior, 0,59 e 0,95; na artéria cerebral média, 0,56 e 0,93; e na artéria cerebral posterior, 0,62 e 0,64 . Foram observadas alterações no IR, em relação ao valor de referência, nas artérias cerebrais anterior e média.
No único caso de neonato com toxoplasmose cerebral houve aumento progressivo do IR, o qual, na artéria cerebral anterior variou de 0,51 a 0,70 , na artéria cerebral média variou de 0,42 a 0,75 , e na artéria cerebral posterior variou de 0,61 a 0,71 , indicando que neste tipo de lesão também ocorreram alterações precoces no IR.

Dentre os 34 casos (68\%), do total de neonatos, nos quais não foram detectadas lesões cerebrais pela USC, 18 (53\%) apresentaram alterações no IR, em 13 (38\%) o Doppler estava normal e em 3 casos (9\%) o Doppler não foi realizado devido ao comprometimento do quadro clínico. Nos casos com Doppler normal ocorreu um óbito e naqueles com Doppler alterado ocorreram três óbitos.

A Tabela 3 apresenta os valores médios do IR para as artérias cerebrais anterior, média e posterior dos 18 neonatos sem lesão cerebral, mas com alterações no Doppler.
Tabela 3 Valores de média e desvio-padrão do IR das artérias cerebrais dos neonatos prematuros sem alteração cerebral pela USC, mas com Doppler alterado $(n=18)$.

\begin{tabular}{ccccc}
\hline Período & Exame (dias) & ACA (M \pm DP) & ACM (M \pm DP) & ACP (M \pm DP) \\
\hline 1 & 1 a 5 & $0,68 \pm 0,08$ & $0,79 \pm 0,06$ & $0,72 \pm 0,10$ \\
2 & 10 a 17 & $0,73 \pm 0,08$ & $0,79 \pm 0,08$ & $0,75 \pm 0,06$ \\
3 & 18 a 30 & $0,75 \pm 0,06$ & $0,77 \pm 0,09$ & $0,73 \pm 0,10$ \\
4 & 30 a 44 & $0,74 \pm 0,10$ & $0,80 \pm 0,10$ & $0,76 \pm 0,07$ \\
\hline
\end{tabular}

ACA, artéria cerebral anterior; ACM, artéria cerebral média; ACP, artéria cerebral posterior; M, média; DP, desviopadrão. 
De acordo com a Tabela 3, apesar de os valores médios do IR estarem dentro dos níveis de normalidade, as alterações ocorridas no IR estavam relacionadas com o valor máximo, que se apresentou superior à normalidade nos períodos 2 a 4 na artéria cerebral anterior $(0,81,0,81$ e 0,84 , respectivamente), nos períodos 1 a 4 na artéria cerebral média $(0,85,0,87,0,86$ e 0,90 , respectivamente) e nos períodos 1 a 4 na artéria cerebral posterior $(0,82,0,81,0,83$ e 0,83 , respectivamente).

Apesar de os valores do IR terem sido pouco acima da normalidade, exceto na artéria cerebral anterior, na qual foi ocorrendo aumento progressivo, foram constatadas alterações no IR mesmo em neonatos sem lesão cerebral aparente à USC.

Os resultados do teste $t$ com correção de Welch mostraram diferença significante entre valores médios de IR das artérias cerebrais anterior e posterior ( $p=0,001$ e $p$ $=0,043$, respectivamente) dos neonatos sem alterações cerebrais mas com Doppler alterado, em relação àqueles com alterações e Doppler alterado, quando realizados no período 1 ( 1 a 5 dias de vida).

\section{Evolução do quadro de lesão cerebral}

Dos 10 neonatos com HIC e Doppler alterado, todos tiveram piora do quadro, com ocorrência de dois óbitos durante o período do estudo. Esta piora se caracterizou também na evolução no grau de HIC.

Dos dois neonatos com LPV e Doppler alterado, todos tiveram piora do quadro, sem ocorrência de óbito durante o período do estudo. Destes, um evoluiu da fase de desenvolvimento de necrose (exame feito entre o $10^{\circ}$ e $17^{\circ}$ dias de vida) para fase cística com lesão do parênquima cerebral (entre 30 e 44 dias), enquanto o outro apresentou hidrocefalia moderada em exame realizado entre o $10^{\circ}$ e $17^{\circ}$ dias de vida, evoluindo para hidrocefalia acentuada entre 30 e 44 dias de vida ${ }^{(\mathbf{1 3})}$.

O único caso de toxoplasmose e Doppler alterado evoluiu para piora do quadro, sem óbito. Pela USC realizada entre o primeiro e quinto dias, foram detectadas calcificações no parênquima cerebral e moderada dilatação do sistema ventricular. Entretanto, em exame feito entre o $18^{\circ}$ e $30^{\circ}$ dias de vida, foi observada acentuada dilatação ventricular bilateral.
Por meio do diagrama de associação por análise de dependência para comparação do prognóstico (piora ou morte) entre Doppler alterado e piora com Doppler normal e não piora, constatou-se que o Doppler alterado não é preditor de morte, mas está relacionado com a piora do quadro hemodinâmico e clínico $(p=0,032)$.

\section{DISCUSSÃO}

Este estudo, por ter sido conduzido em prematuros de 29 a 32 semanas e com peso ao nascimento entre 550 e $1.500 \mathrm{~g}$, com o intuito de avaliar a hemodinâmica cerebral, reforça sua importância devido à dificuldade em examiná-los e manuseá-los em fase tão frágil de suas vidas.

A USC realizada nos neonatos prematuros de muito baixo peso mostrou-se útil na detecção de alterações cerebrais como HIC, LPV e toxoplasmose em $32 \%$ dos casos do presente estudo. Os resultados demonstram que a aplicação clínica deste método de diagnóstico por imagem é de grande valia na avaliação do prematuro, especialmente se realizado até o quinto dia de vida, pois fornece novas informações, contribuindo para melhor conduta terapêutica e desenvolvimento neurológico desses pacientes pediátricos. Além disso, medidas de suporte vitais aplicadas nesses prematuros, o quanto antes possível, são essenciais para evitar piora nas lesões hipóxicoisquêmicas e infecciosas, assim como possibilitar acompanhamento evolutivo ${ }^{(\mathbf{1 4})}$.

No presente estudo, a aplicação do Doppler não detectou alteração no IR em 13 neonatos prematuros (38\%) sem lesões cerebrais, e em 18 neonatos prematuros (53\%) sem lesão cerebral houve alteração no IR. O valor médio do IR da artéria cerebral anterior foi de 0,72 no exame realizado entre o primeiro e quinto dias de vida. Em estudo conduzido em 121 neonatos prematuros e a termo com peso entre $1.070 \mathrm{e}$ $3.750 \mathrm{~g}$, foi encontrado IR médio de 0,73 em ambas as artérias cerebrais anteriores $^{(15)}$. Entretanto, no referido estudo ${ }^{(15)}$, além de a amostra ter sido composta de neonatos prematuros e a termo e o peso ter sido diferente, não houve menção da época, em dias, da realização do exame, dificultando a comparação pela diferença das variáveis analisadas nos estudos, apesar de ambos terem apresentado o IR dentro dos níveis de normalidade.

A relação entre velocidade do fluxo cerebral e lesões cerebrais tem sido confirmada em diversos estudos ${ }^{(\mathbf{1 6 , 1 7})}$. Flutuações no fluxo cerebral observadas em neonatos prematuros decorrentes de expansão rápida de volume podem contribuir para hemorragia intraventricular ${ }^{(\mathbf{1 8 , 1 9 )}}$. A hipercarbia aguda tem um maior impacto no fluxo cerebral pelo relaxamento da vasculatura, aumentando, assim, o fluxo sanguíneo, podendo ser esse um dos componentes na patogênese da HIC. Em contraste, a hipocarbia está relacionada à diminuição do fluxo e à $\mathrm{LPV}^{(\mathbf{2 0 )}}$. O baixo fluxo leva à isquemia, a qual resulta em morte tecidual ou uma subsequente reperfusão, que pode causar ruptura de vasos com surgimento de leucomalácia ${ }^{(21)}$. A HIC com subsequente dilatação intraventricular está associada a um risco maior de leucomalácia cística ${ }^{(22)}$.

HICs ocorrem mais frequentemente na primeira semana de vida e $97 \%$ dos casos são detectados pela USC ${ }^{(\mathbf{2 3})}$. No presente estudo, os exames foram realizados logo nos primeiros cinco dias de vida, encontrando alterações de imagem e do IR neste período. Em estudo realizado em neonatos prematuros com idade entre 28 e 36 semanas e peso entre 720 e $2.530 \mathrm{~g}$, foi observado, no sétimo dia de vida, IR mais elevado nos prematuros sem lesão cerebral quando comparados aos com HIC ${ }^{(24)}$. Essas alterações encontradas foram semelhantes às do presente estudo, exceto que neste o diagnóstico foi feito antes do sétimo dia de vida. O IR abaixo dos valores de normalidade explica a maior acentuação na evolução da hemorragia cerebral.

USC com Doppler foi realizada nos três primeiros dias de vida em estudo em 51 neonatos prematuros com peso abaixo de $1.751 \mathrm{~g}^{\mathbf{( 2 5 )}}$. Nesse estudo foi determinado o IR na artéria cerebral anterior, sendo comparados os valores para essa artéria nos hemisférios cerebrais direito e esquerdo em grupos distintos de neonatos (sem HIC, com HIC unilateral e com HIC bilateral), sendo observado que o coeficiente de variação do lado da hemorragia não diferiu significantemente daquele do lado não afetado ${ }^{(25)}$. No presente estudo, não houve preocupação com o lado a ser examinado nem com a pressão do transdutor sobre a 
fontanela, a fim de se evitar ao máximo a movimentação do neonato. Com a movimentação do prematuro, pode ocorrer aumento da frequência cardíaca e redução artificial do IR, pois a velocidade diastólica é medida na fase média da diástole, quando a velocidade é maior ${ }^{(6)}$. Já a pressão do transdutor sobre a fontanela pode aumentar transitoriamente a pressão intracraniana, reduzindo o fluxo durante a diástole e aumentando o $\mathrm{IR}^{(\boldsymbol{6})}$.

É possível que o aumento encontrado, no presente estudo, para o valor médio do IR nas artérias cerebrais anterior, média e posterior dos neonatos com HIC se deva à evolução em graus da hemorragia nos neonatos com HIC. Este resultado está em concordância com outro estudo ${ }^{(26)}$ realizado em 57 neonatos com idade gestacional $<37$ semanas durante os primeiros dez dias de vida, o qual não constatou diferença entre o valor de IR nas artérias cerebrais anterior e média nos neonatos com HIC leve (graus I e II), quando comparado com prematuros sem lesão. Por outro lado, os prematuros com HIC moderada e grave (graus III e IV, respectivamente) apresentaram valor do IR significantemente maior em relação aos neonatos prematuros sem lesão. De acordo com o estudo acima citado, os achados sugerem que alterações na resistência vascular cerebral ocorrem com o desenvolvimento ou como consequência de lesões hipóxicoisquêmicas no recém-nascido. Deve-se ressaltar que no referido estudo não foram informados os valores médios do IR, tanto para HIC leve como para HIC moderada e grave, o peso dos neonatos e a idade gestacional mínima destes.

Quanto aos casos de LPV encontrados no nosso estudo, houve alteração no valor superior do IR em 50\% dos casos na USC com Doppler realizada entre o primeiro e quinto dias de vida. Em estudo de investigação do IR apenas da artéria cerebral anterior, nas primeiras 72 horas de vida, em 53 neonatos com idade média gestacional de 30,4 semanas e peso inferior a $1.500 \mathrm{~g}$, foram observadas lesão da substância branca cerebral e alteração do valor máximo do IR, mas em 5,6\% dos neonatos ${ }^{(\mathbf{1 4})}$. Essa investigação se deu em neonatos com idade gestacional e peso semelhantes aos do presente estudo, mas o Doppler foi realizado em apenas uma artéria, sendo encontrada preva- lência menor que a do nosso estudo. A discrepância nos resultados pode ser devida ao fato de o presente estudo ter avaliado neonatos com pesos extremos, como $550 \mathrm{~g}$, e feito Doppler nas três artérias cerebrais.

O IR alterado está associado com complicações na evolução da lesão da substância branca cerebral, sendo, portanto, importante parâmetro a ser avaliado em neonatos. Este tipo de lesão pode causar sequelas neuropsicomotoras graves e levar a paralisia cerebral e/ou déficit cognitivo e comportamental $^{(27,28)}$. O diagnóstico precoce e uma estratégia terapêutica adequada podem minimizar sequelas causadas por essas lesões ${ }^{(4)}$.

No presente estudo foi constatada piora da LPV, a qual apresentou dois tipos de evolução na imagem: 1) da fase de desenvolvimento de necrose para fase cística com lesão do parênquima cerebral; 2) de hidrocefalia moderada para acentuada, de acordo com a classificação da hidrocefalia ${ }^{(13)}$, estando estes resultados em concordância com os da literatura ${ }^{(27,28)}$.

O diagnóstico de toxoplasmose à USC no presente estudo foi obtido pela detecção de calcificações no parênquima cerebral e moderada dilatação do sistema ventricular. Conforme descrito na literatura, tanto a calcificação no parênquima como a dilatação ventricular detectadas pelo exame ultrassonográfico são características de infecções intracranianas ocasionadas pela toxoplasmose ${ }^{(29)}$. Exames laboratoriais confirmaram o diagnóstico ultrassonográfico de toxoplasmose no paciente do nosso estudo.

Considerando que a sobrevida de neonatos com menos de $1.000 \mathrm{~g}$ é cada vez maior e o número de prematuros aumentou consideravelmente nos últimos anos, tornando a HIC e a LPV doenças de prevalência significativa entre os mesmos ${ }^{(29)}$, estudos como este são instrumentos de predição do risco no período neonatal para alterações no território vascular cerebral correspondente e o prognóstico neurológico em médio e longo prazos.

A ultrassonografia com Doppler possui vantagens, como baixo custo econômico, versatilidade e mobilidade do aparelho, tornando factível o exame à beira do leito, além de inocuidade e ausência de emissão de radiação ${ }^{(30)}$, que permitem que o exame seja repetido várias vezes sem a necessi- dade de sedação do neonato ${ }^{(\mathbf{3 1})}$. Todavia, este método apresenta desvantagens, como menor sensibilidade e especificidade para lesões parenquimatosas, principalmente em alterações isquêmicas, em relação à tomografia e à ressonância magnética. A ressonância é mais sensível que a USC e a tomografia na avaliação da extensão de lesões da substância branca, porque pode detectar áreas de desmielinização e formação de cicatrizes gliais ${ }^{(32)}$. De acordo com a literatura, não existe ainda indicação de ressonância como rotina em exames de USC alterados ${ }^{(\mathbf{3 1})}$, o que poderia ser conduta adequada nesses casos.

Todos os dados obtidos durante a presente investigação permitem considerar que a mensuração seriada do IR das artérias intracranianas, utilizada na técnica com Doppler, registra de maneira segura modificações na circulação cerebral do neonato prematuro, inclusive naqueles nos quais não foram detectadas alterações nas imagens. Ao permitir a observação destas modificações e flutuações na velocidade do fluxo sanguíneo cerebral em neonatos com determinadas enfermidades que predispõem à ocorrência de hemorragia cerebral, tais como síndrome da angústia respiratória, aumentos na pressão venosa central e pressão arterial média, a análise do IR possibilita ao neonatologista instituir medidas terapêuticas que reduzam estas ocorrências ou minimizem a sua gravidade ${ }^{(24)}$.

\section{CONCLUSÕES}

A USC em neonatos prematuros de muito baixo peso realizada precocemente é importante na detecção de lesões cerebrais e no prognóstico.

A utilização da USC com Doppler é importante no monitoramento de alterações na hemodinâmica cerebral, devendo ser usada para o diagnóstico precoce de hemorragias e lesões hipóxico-isquêmicas.

Existe correlação entre a presença de alterações na hemodinâmica cerebral e subsequente desenvolvimento de hemorragias e lesões hipóxico-isquêmicas, por meio da mensuração do IR.

Alterações do IR, embora não preditoras de morte, estão relacionadas com a gravidade do quadro clínico em neonatos prematuros de muito baixo peso. 


\section{REFERÊNCIAS}

1. Rades E, Bittar RE, Zugaib M. Determinantes diretos do parto prematuro eletivo e os resultados neonatais. Rev Bras Ginecol Obstet. 2004;26: 655-62.

2. Rumack CM, Drose JA. Exame cerebral do neonato e do lactente. In: Rumack CM, Wilson SR, Charboneau JW, editores. Tratado de ultra-sonografia diagnóstica. $3^{\mathrm{a}}$ ed. Rio de Janeiro, RJ: Elsevier; 2006. p. 1623-701.

3. Abrão N, Moreira MT, Amaro Júnior E. Afecções hemorrágicas e hipóxico-isquêmicas. In: Abrão N, Amaro Júnior E, Cerri GG, editores. Ultra-sonografia intracraniana: anatomia ultra-sonográfica, afecções hemorrágicas e hipóxico-isquêmicas. São Paulo, SP; Sarvier; 1998. p. 107-24.

4. Silveira RC, Procianoy RS. Lesões isquêmicas cerebrais no recém-nascido pré-termo de muito baixo peso. J Pediatr (Rio J). 2005;81(1 Supl): S23-32.

5. Papile LA, Burstein J, Burstein R, et al. Incidence and evolution of subependymal and intraventricular hemorrhage: a study of infants with birth weights less than 1,500 gm. J Pediatr. 1978;92: 529-34.

6. Taylor GA. Doppler do cérebro no neonato e do lactente. In: Rumack CM, Wilson SR, Charboneau JW, editores. Tratado de ultra-sonografia diagnóstica. $3^{\mathrm{a}}$ ed. Rio de Janeiro, RJ: Elsevier; 2006. p. 1703-22.

7. Archer LN, Evans DH, Levene MI. Doppler ultrasound examination of the anterior cerebral arteries of normal newborn infants: the effect of postnatal age. Early Hum Dev. 1985;10:255-60.

8. Liu J, Cao HY, Huang XH, et al. The pattern and early diagnostic value of Doppler ultrasound for neonatal hypoxic-ischemic encephalopathy. J Trop Pediatr. 2007;53:351-4.

9. Vollmer B, Roth S, Baudin J, et al. Predictors of long-term outcome in very preterm infants: gestational age versus neonatal cranial ultrasound. Pediatrics. 2003;112:1108-14.

10. De Vries LS, Van Haastert IL, Rademaker KJ, et al. Ultrasound abnormalities preceding cerebral palsy in high-risk preterm infants. J Pediatr. 2004;144:815-20.

11. Bland M. An introduction to medical statistics. 3rd. ed. Oxford, UK: Oxford University Press; 2002.

12. Cordeiro JA. Analysis of dependency. Relatório técnico 48/87. Campinas, SP: Instituto de Matemática - Unicamp; 1987.

13. Garrett WJ, Kossoff G, Warren PS. Cerebral ventricular size in children: a two-dimensional ultrasonic study. Radiology. 1980;136:711-5.

14. Argollo N, Lessa I, Ribeiro S. Medidas do índice de resistência ao Doppler craniano em recém-nascidos pré-termo com lesão da substância branca cerebral. J Pediatr (Rio J). 2006;82:221-6.

15. Deeg KH, Rupprecht T. Pulsed Doppler sonographic measurement of normal values for the flow velocities in the intracranial arteries of healthy newborns. Pediatr Radiol. 1989;19:71-8.

16. Mainous RO, Looney S. A pilot study of changes in cerebral blood flow velocity, resistance, and vital signs following a painful stimulus in the premature infant. Adv Neonatal Care. 2007;7:88-104

17. Pellicer A, Valverde E, Gayá F, et al. Postnatal adaptation of brain circulation in preterm infants. Pediatr Neurol. 2001;24:103-9.

18. Wildrick D. Intraventricular hemorrhage and long-term outcome in the premature infant. J Neurosci Nurs.1997;29:281-9.

19. Ment LR, Stewart WB, Ardito TA, et al. Germinal matrix microvascular maturation correlates inversely with the risk period for neonatal intraventricular hemorrhage. Dev Brain Res. 1995;84: 142-9.

20. Bell PL, Ellerbee S. Impaired cerebral vascular blood flow in the premature infant. J Perinat Neonatal Nurs. 1993;7:49-55.

21. Verma U, Tejani N, Klein S, et al. Obstetric antecedents of intraventricular hemorrhage and periventricular leukomalacia in the low-birthweight neonate. Am J Obstet Gynecol. 1997; 176:275-81.

22. Larroque B, Marret S, Ancel PY, et al. White matter damage and intraventricular hemorrhage in very preterm infants: the EPIPAGE study. J Pediatr. 2003;143:477-83.

23. Bittar RE, Zugaib M. Parto prematuro: fatores predisponentes e prevenção. In: Marcondes E, Costa Vaz FA, Araujo Ramos JL, organizadores. Pediatria básica. $9^{\mathrm{a}}$ ed. São Paulo, SP: Sarvier; 2003. p. 337-45.

24. Assis MC, Machado HR. Ecografia transfontanelar com fluxo a cores em recém-nascidos prematuros. Arq Neuropsiquiatr. 2004;62:68-74.

25. Kuban KC, Skouteli H, Cherer A, et al. Hemorrhage, phenobarbital, and fluctuating cerebral blood flow velocity in the neonate. Pediatrics. 1988;82:548-53.

26. Mires GJ, Patel NB, Forsyth JS, et al. Neonatal cerebral Doppler flow velocity waveforms in the pre-term infant with cerebral pathology. Early Hum Dev. 1994;36:213-22.

27. Fukuda S, Kuwabara S, Yasuda M, et al. Hemodynamics of the posterior cerebral arteries in neonates with periventricular leukomalacia. J Clin Ultrasound. 2005;33:24-8.

28. Kubota T, Okumura A, Hayakawa F, et al. Combination of neonatal electroencephalography and ultrasonography: sensitive means of early diagnosis of periventricular leukomalacia. Brain Dev. 2002;24:698-702.

29. Plese JPP, Nakagawa GE, Olivi GGB, et al. Hemorragia intracraniana peri e intraventricular. In Marcondes E, Costa Vaz FA, Araujo Ramos JL, organizadores. Pediatria básica. $9^{\mathrm{a}}$ ed. São Paulo, SP: Sarvier; 2003. p. 574-8.

30. Farage L, Assis MC. Achados ultra-sonográficos da hemorragia intracraniana em recém-nascidos prematuros. Arq Neuropsiquiatr. 2005;63:814-6.

31. Ment LR, Bada HS, Barnes P, et al. Practice parameter: neuroimaging of the neonate: report of the Quality Standards Subcommittee of the American Academy of Neurology and the Practice Committee of the Child Neurology Society. Neurology. 2002;58:1726-38.

32. Siegel MJ. Cérebro. In: Siegel MJ, editor. Ultrasonografia pediátrica. $3^{\mathrm{a}}$ ed. Rio de Janeiro, RJ: Guanabara Koogan; 2003. p. 37-108. 